\title{
EVALUACION POST - CAPTURA DE Aotus vociferans y Aotus nancymae EN BOSQUES DE LA AMAZONIA PERUANA
}

Rolando A quino, Pablo Puertas y Filomeno Encarnación (*)

\section{RESUMEN}

A fin de evaluar los efectos de la remoción parcial de las poblaciones de Aotus vociferan y A. nancymae, hicimos capturas en áreas correspondientes a los bosques de bajial de las cuencas de los ríos $\mathrm{N}$ apo y A mazonas.

En la primera captura de A. vociferans ejecutada en 1986 en el río Tamboryacu, fueron removidas 35.5\% de la población y en 1989 en el río Santa M aría el $55 \%$ de la población. Durante la evaluación de 1991, en el río Tamboryacu el incremento de la población remanente alcanzó el $144.0 \%$ y el $93.0 \%$ del nivel de precaptura de 1986, mientras que en el río Santa M aría, el incremento alcanzó el 12.5\% de la población remanente y el 50\% del nivel de pre-captura de 1989.

En referencia a A. nancymae, durante la primera captura ejecutada en 1989 en el río Tahuayo fueron removidas el $50.0 \%$ de la población y en la quebrada $Y$ anayacu el $59.0 \%$ de la población. Dos años después, el incremento en el río Tahuayo fue el $10.0 \%$ de la población remanente, alcanzando el $48.0 \%$ del nivel de pre-captura de 1989 , mientras que en la quebrada $Y$ anayacu el incremento fue de $55.0 \%$ de la población remanente y $61.0 \%$ del nivel de pre-captura de 1989.

El bajo nivel de recuperación de la población de A. nancymae en el río Tahuayo tiene relación con la intensa caza para subsistencia yen menor grado con la deforestación. En el río Santa M aría, se debe únicamente a la deforestación con fines agrícolas, que en 1991 alcanzó el 51.0\% del área total.

Después de las capturas, los nacimientos y las inmigraciones tanto en $A$. vociferans como en A. nancymae contribuyeron al repoblamiento en las áreas afectadas, cuyas proporciones no fue posible precisarlos.

\section{INTRODUCCION}

Entre los primates neotropicales, Aotus nancymae y A. vociferans son algunas especies de este género que tienen amplio uso en los laboratorios para las investigaciones en malaria y otras relacionadas a la conducta del hombre. Debido a esta importancia.

Instituto V eterinario de Investigaciones Tropicales y de A Itura- IV ITAP.O Box 575 Iquitos-Perú U niversidad $N$ acional M ayor de San M arcos 
biomédica, el Gobierno Peruano en contribución a la salud mundial transfirió desde 1981 una cantidad razonable de estos primates, todos provenientes de las áreas naturales. Ultimamente para las capturas se definieron las Ilamadas áreas de manejo, de donde fueron removidas cierto número de ejemplares con miras a desarrollar un seguimiento sobre la capacidad de recuperación. Tales informaciones podrían contribuir a la conservación y el manejo, especialmente en áreas donde las poblaciones y el hábitat son afectados como consecuencia de las capturas.

En tal sentido desde 1986 a 1991, en áreas limitadas de los ríos Tamboryacu y Santa M aría en la cuenca del río N apo y río Tahuayo y quebrada $\mathrm{Y}$ anayacu en la cuenca del río A mazonas hemos ejecutados capturas. Los resultados de la densidad pre-captura y post-captura son presentados aquí para evaluar los efectos causados por la remosión parcial o casi total de la población.

\section{MATERIALES Y METODOS}

\section{AREAS DE ESTUDIO}

Las áreas de estudio para A. vociferans, se encuentran en el curso bajo de los ríos Tamboryacu y Santa M aría, ambos afluentes derecho del río Napo (Fig. 1)

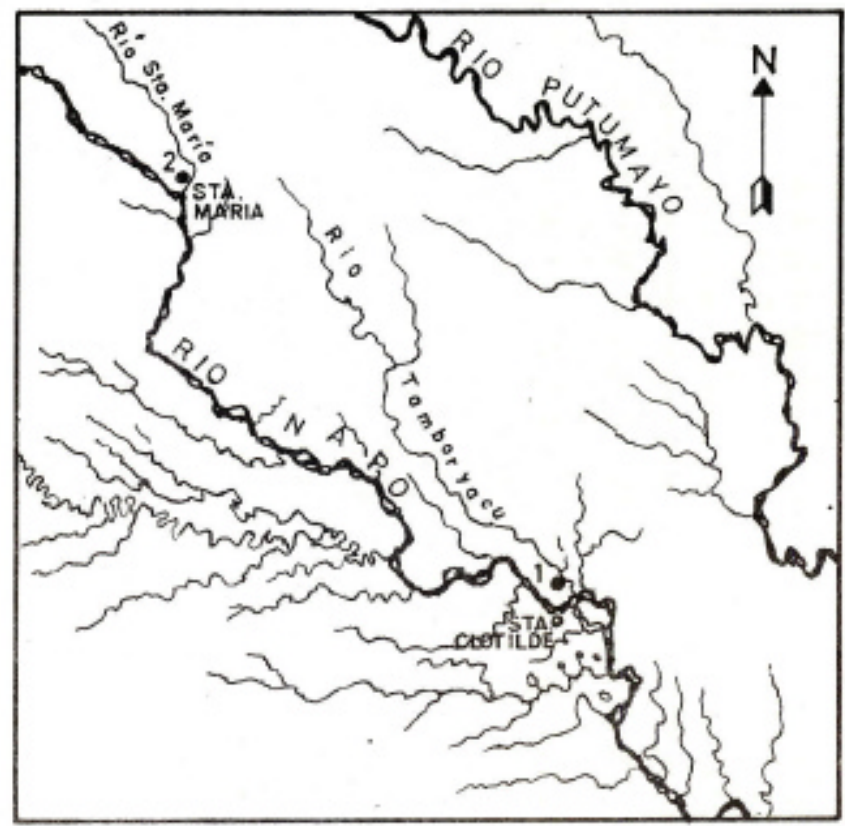


Para A . namcymae están ubicados en el curso bajo del río Tahuayo y quebrada de $Y$ anayacu, ambos afluentes del río A mazonas (Fig. 2)

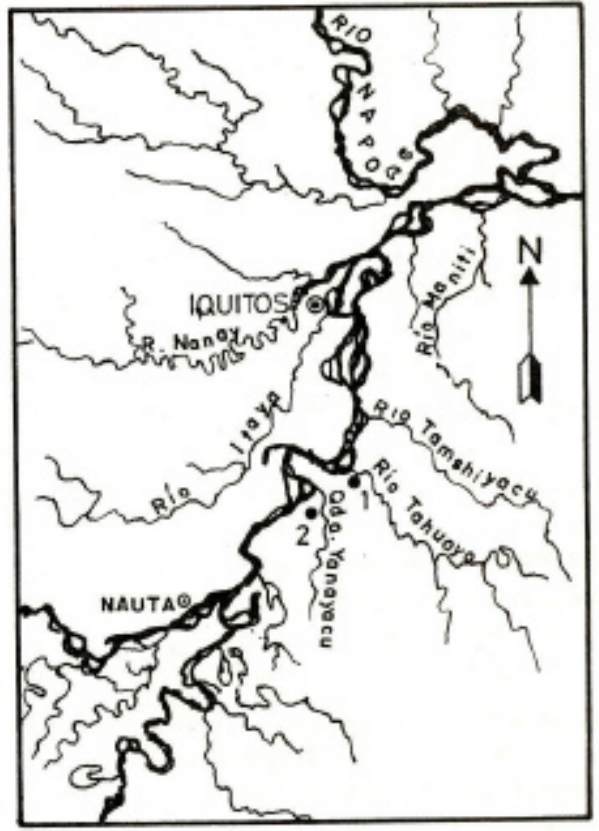

En las áreas de estudio existe variada arquitectura vegetal, muchos tipos de suelos y variables efectos periódicos estacionales de los flujos de masas de agua y diversas actividades humanas.

En los bosques de Tamboryacu, el suelo es limoso-arcilloso, de origen aluvial antiguo, de relieve plano e inundado por agua negra, cuyo bosque del tipo 'tahuampa' (Encarnación, 1985) está representado por Naucopsis imitans tamamuri, Eschweilera sp. "machimango, Calycophyllum spruceanum "capirona, Ficus spp. "renaco", Manilkara sp. 'quinilla, Perinari parili 'parinari, entre otros; que son de gran porte pero sin valor comercial. A quí el sotobosque es disperso, mientras que los demás estratos soportan pocos enmarañados de bejucos y epífitos.

En Santa María, el suelo es limoso, de origen aluvial reciente, ligeramente ondulado e inundado temporalmente por agua blanca o mezcla con agua negra. EI bosque es del tipo tahuampa (Encarnación, 1985), representado por Calycophyllum spruceanum "capirona", Naucleopsis glabra 'tamamuri", N. imitans "tamamuri, Scheelea sp. shapaja", Ficus sp. renaco, en asociaciones denominadas capironales", “tamurales, shapajales' y renacales', respectivamente. Estas especies son de gran altura pero sin valor comercial. El sotobosque está formado por arbustos delgados y erguidos, sin aspecto de motorrales, el estrato medio alcanza hasta $20 \mathrm{~m}$. de altura, el estrato 
superior hasta $30 \mathrm{~m}$., con escasos bejucos y enredaderas y los árboles emergentes que son pocos sobrepasan los $40 \mathrm{~m}$. de altura.

En los bosques correspondientes al río Tahuayo, el suelo es limoso y de origen aluvial reciente, de relieve plano e inundado por agua negra, mezclas y blancas (Sioli, 1974; Encarnación 1985) sucesivamente en un periodo de 'creciente'. Estos caracteres determinan el bosque de “Tahuampa' (Encarnación, 1985), representado por Hura crepitans "catahua", Couroupita subsessillis "ayahuma", Clarisia biflora "capinuri" Chorisia insignis "lupuna", Ceiba samauma "huimba", Campsiandra spp. "huacapurana y Macrolobiumacaciaefolium "pashaco", entre otros. Las especies mencionadas superan los $25 \mathrm{~m}$. de altura y son de importancia maderable, por consiguiente la estructura y cubierta vegetal del bosque han quedado alteradas por la tala selectiva. Sin embargo, aun podemos distinguir un sotobosque tupido y enmarañado del tipo matorral, un estrato inferior hasta los $8 \mathrm{~m}$. de altura un estrato medio hasta los 15 $\mathrm{m}$. de altura con árboles delgados y abundantes bejucos y enredaderas, un estrato superior hasta los $25 \mathrm{~m}$. de altura, con árboles maduros, muchos de ellos decaídos y senescentes y cubiertos de epifitos y hemiepifitos y algunos árboles emergentes con al tura hasta de $40 \mathrm{~m}$.

En los bosques de $Y$ anayacu, el suelo es igualmente limoso y de origen aluvial reciente, el relieve plano e inundado por aguas negras y de mezcla con agua blanca en periodo de "creciente" (Sioli, 1974; Encarnación 1985), que determinan igualmente un típico bosque de "tahuampa", con una alta predominancia de lagunas. La composición florística es muy similar al de Tahuayo, aun cuando en este bosque predominan además de las especies citadas anteriormente Calycophllum spruceanum y Eschweilera spp. A quí igualmente se advierten los mismos estratos considerados para el Tahuayo.

$L$ as evaluaciones post-captura en Santa M aría, Tahuayo y $Y$ anayacu se hicieron empleando la modalidad de ubicación de los grupos familiares, mientras que en Tamboryacu por ubicación y captura de la mayor parte de los grupos familiares.

De las áreas citadas, a excepción de Tamboryacu, las demás presentan una fuerte alteración como consecuencia de la extracción forestal selectiva y deforestación con fines agrícolas.

\section{METODOS}

L as evaluaciones pre-captura fueron ejecutadas en 1986 en Tamboryacu en un área de $0.6 \mathrm{~km} 2$, en Y anayacu y Santa María en 1989 en un área de $1.0 \mathrm{~km}^{2}$ y en $Y$ anayacu en 1989 en un área de $2.0 \mathrm{~km}^{2}$.

Los métodos para la ubicación y captura de los grupos familiares fueron los mismos que se vienen empleando desde 1981 y se encuentran descritas en Aquino y Encarnación (1986). Sin embargo, la mayoría de los grupos fueron capturados mediante el método de aislamiento del árbol de dormir.

En Tahuayo, las capturas y ubicación de los grupos familiares fueron realizadas entre Marzo y Abril, coincidente con el periodo de 'creciente. En consecuencia, toda 
actividad relacionada a la ubicación y captura fueron facilitadas mediante el uso de canoas. En Y anayacu, Tamboryacu y Santa M aría, estas actividades fueron realizadas entre Noviembre y Diciembre coincidente con el periodo de "vaciante", por lo tanto las actividades para la ubicación y captura se realizaron a pie firme. La ubicación y captura de los individuos de Aotus fueron facilitadas con la ayuda de guías y operarios experimentados y conocedores del lugar. En Tahuayo, las capturas y registros de grupos familiares incluyeron únicamente a todos aquellos localizados desde la ribera hasta los $250 \mathrm{~m}$. hacia el interior del bosque en un tramo de $4 \mathrm{~km}$. En yanayacu y Tamboryacu en un tramo de $2 \mathrm{~km}$. e igual amplitud. En Santa M aría, el área comprendió una faja de $1.0 \mathrm{~km}^{2}$ situada entre los ríos Santa maría y $\mathrm{Napo}$. En todas las áreas, tanto para las evaluaciones pre-captura y post-captura fueron aperturadas trochas paralelas y perpendiculares distantes en $200 \mathrm{~m}$. entre una y otra trocha, las mismas que facilitaron la ubicación de los grupos.

\section{RESULTADOS}

\section{TAMAÑO DE GRUPO}

El tamaño de los grupos registrados durante y después de las cosechas para A. vociferans se presenta en la Tabla 1. En Tamboryacu los tamaños fluctuaron entre dos y cuatro en 1986 y entre tres y cuatro en 1991, con promedios muy similares, los mismos que coinciden con los registrados por A quino et al. (1990) para esta especie. En Santa María, el tamaño de los grupos variaron entre dos y cinco en 1989 y entre dos y cuatro en 1991, con promedios de 3.85 y 3.37 individuos por grupo, respectivamente. Los promedios registrados tanto en Tamboryacu y santa maría no parecen diferir significativamente.

La frecuencia del tamaño en 1991 en Tamboryacu y Santa María fueron los mismos a los registrados en 1986 y 1989, donde grupos de cuatro individuos predominaron, coincidiendo así con los registrados por A quino et al. (1990) para los meses de octubre y diciembre. El tamaño de los grupos registrados durante y después de las capturas para A. mancymae se muestra en la Tabla 2. En el río Tahuayo, los tamaños fluctuaron entre dos y cinco, tanto en 1989 como en 1991, con promedios de 3.86 y 3.55 por grupo, respectivamente, los mismos que son cercanos a los registrados por Aquino et al. (1990). En $Y$ anayacu los tamaños para esta misma especie fluctuaron entre dos y seis en 1989 y entre dos y cuatro en 1991, con promedios de 4.3. y 3.4 por grupo, respectivamente. En Tahuayo, los promedios no parecen diferir significativamente, mientras que en $Y$ anayacu la diferencia podría ser significativa.

En cuanto a la frecuencia del tamaño de grupos, en Tahuayo no hubo mayor variación, por cuanto grupos de cuatro individuos fueron los más frecuentes tanto en 1989 como en 1991. En Y anayacu, las frecuencias de los tamaños. 
Registrados en 1991. En Y anayacu, las frecuencias de los tamaños registrados en 1991 fueron diferentes a los de 1991 grupos de dos individuos prevalecieron, contrastando con la de 1989 y otros registros (A quino y Encarnación, 1986 A quino et al. 1990) donde grupos de cuatro individuos predominaron y que representaron el $50 \%$ de los grupos.

TABLA 1: Tamaños de grupos registrados en la población de Aotus vociferans en las áreas de estudio de los ríos Tamboryacu y Santa María

\begin{tabular}{|c|c|c|c|c|}
\hline \multirow{2}{*}{ Tamaño } & \multicolumn{2}{|l|}{ TAMBORYACU } & \multicolumn{2}{|c|}{ SANTA MARIA } \\
\hline & $\begin{array}{l}1986 \\
\text { Frecuencia \% }\end{array}$ & $\begin{array}{l}1991 \\
\text { Frecuencia \% }\end{array}$ & $\begin{array}{l}1989 \\
\text { Frecuencia \% }\end{array}$ & $\begin{array}{l}1991 \\
\text { Frecuencia \% }\end{array}$ \\
\hline 2 & 222.2 & 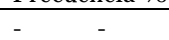 & $\begin{array}{ll}1 & 7.2\end{array}$ & $1 \quad 12.5$ \\
\hline 3 & $5 \quad 55.6$ & $10 \quad 77.0$ & 21. & 37.5 \\
\hline 4 & 22.2 & $3 \quad 23.0$ & 50.0 & 50.0 \\
\hline 5 & & - & 21.4 & - \\
\hline $\begin{array}{l}\text { Total } \\
\text { Promedio } \\
\pm \mathrm{DE}\end{array}$ & $\begin{array}{lc}9 & 100.0 \\
3.0 \pm 0.65\end{array}$ & $\begin{array}{ll}13 & 100.0 \\
3.2 \pm & 0.44\end{array}$ & $\begin{array}{lc}14 & 100.0 \\
3.85 \pm 0.86\end{array}$ & $\begin{array}{l}8 \quad 100.0 \\
3.37 \pm 0.74\end{array}$ \\
\hline
\end{tabular}

TABLA 2. Tamaños de grupos registrados en la población de Aotus nancymae en las áreas de estudio del río Tahuayo y Q uebrada Y anayacu.

\begin{tabular}{|c|c|c|c|c|c|}
\hline \multirow[b]{2}{*}{ Tamaño } & \multicolumn{2}{|c|}{ TAMBORYACU } & \multicolumn{3}{|c|}{ SANTA MARIA } \\
\hline & $\begin{array}{l}1989 \\
\text { Frecuencia \% }\end{array}$ & $\begin{array}{l}1991 \\
\text { Frecuencia \% }\end{array}$ & $\begin{array}{l}1989 \\
\text { Frecuencia \% }\end{array}$ & $\begin{array}{l}19 \\
\mathrm{Fr}\end{array}$ & encia \% \\
\hline 2 & 16.7 & 11.0 & 15.0 & - & - \\
\hline 3 & 213.3 & 33.0 & 15.0 & 6 & 60.0 \\
\hline 4 & $10 \quad 66.7$ & 45.0 & 15.0 & 4 & 40.0 \\
\hline 5 & 213.3 & 11.0 & 31.0 & - & - \\
\hline 6 & - & - & - & - & - \\
\hline $\begin{array}{l}\text { Total } \\
\text { Promedio } \\
\pm \mathrm{DE}\end{array}$ & $\begin{array}{ll}15 & 100.0 \\
3.86 \pm 0.74\end{array}$ & $\begin{array}{l}9 \quad 100.0 \\
3.55 \pm 0.88\end{array}$ & $\begin{array}{l}13 \quad 99.0 \\
4.3 \pm 1.42\end{array}$ & 10 & 100.0 \\
\hline
\end{tabular}

\section{DENSIDAD POBLACIONAL Y RECUPERACIÓN}

Los estimados de la densidad poblacional para A. vociferans y A. nancymae antes y después de las capturas están contenidas en las Tablas 3 y 4 . L os resultados concernientes a la pre-captura fueron obtenidos por localización de los grupos y captura de la mayoría de ellos que habitaron en las áreas de estudio. Las densidades postcaptura en Santa María, Tahuayo y $\mathrm{Y}$ anayacu fueron obtenidos únicamente por localización, mientras que en Tamboryacu por localización y captura de la mayoría de los grupos. En Tamboryacu, durante la primera captura realizada en 1986, 35.5\% de la población fue removida, quedando 29 individuos, la mayoría agrupados en grupos incompletos entre dosy tres individuos. En 1991 la población se incrementó en 144\% de la población remanente, alcanzando el 93.3\% del nivel de la precaptura de 1986. 
En Santa M aría, en la captura de 1989, el 55\% de la población fue removida, quedando como remanentes 24 individuos, de las cuales 16 conformaban grupos completos entre dos y cuatro individuos. Dos años después la población remanente se incrementó en apenas $12.5 \%$, alcanzado así el 50\% del nivel de pre-captura registrada en 1989. En el río Tahuayo, después de la captura realizada en $1989,50 \%$ de la población fue removida, quedando 29 individuos, de las cuales siete fueron grupos completos entre dos y cinco individuos, por lo tanto incluía siete parejas reproductivas. En 1991, es decir, dos años después de la captura, la población se había incrementado en apenas $10 \%$ de la población remanente, habiendo alcanzado únicamente el $55 \%$ del nivel de pre-captura registrado en 1989.

En la quebrada $Y$ anayacu, en la captura realizada en 1989 fueron removidos el $59 \%$ de la población, quedando 23 individuos, la mayoría formando cinco grupos completos entre dos y cinco individuos. Dos años después el incremento fue de $48 \%$ de la población remanente, alcanzando el 61\% del nivel de precaptura registrado en 1989.

\section{TABLA 3.- Densidad poblacional y recuperación de Aotus vociferans en las áreas de estudio de los ríos T amboryacu y Santa M aría}

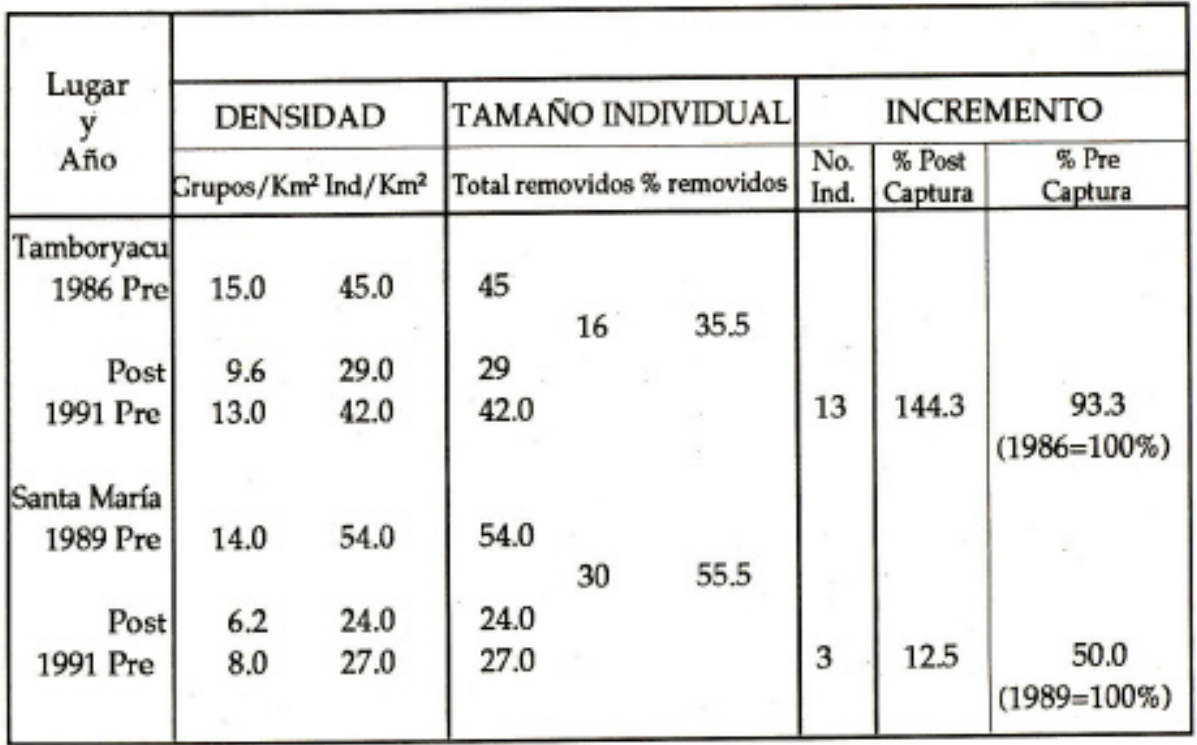


TABL A 4: Densidad poblacional y recuperación de A otus nancymae en las áreas de estudio del río T ahuayo Q uebrada Y anayacu.

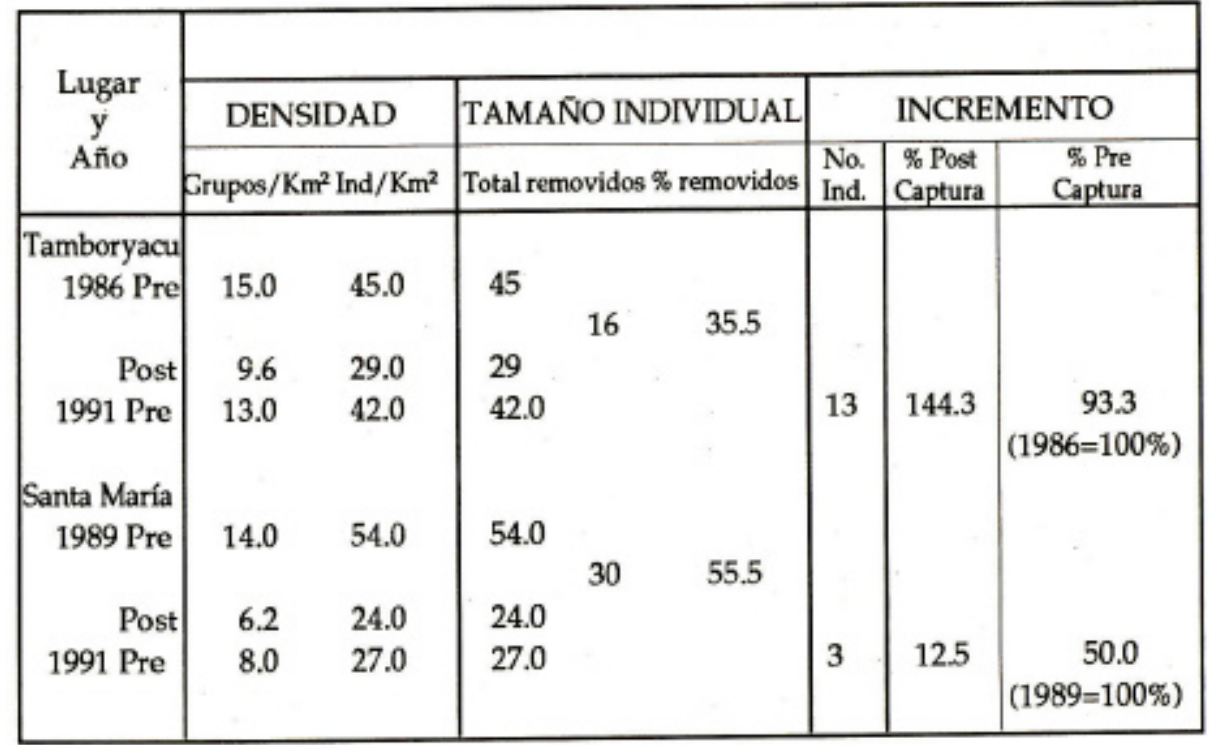

\section{IMPACTO DE LA ACTIVIDAD HUMANA SOBRE LA POBLACIÓN DE AOTUS}

De las cuatro áreas consideradas para el estudio, únicamente en $Y$ anayacu no hubo deforestación con fines agrícolas, pero hubo extracción forestal. En las demás, la actividad agrícola se incrementó tal como podemos apreciar en la Tabla 5. Entre las áreas de estudio, la más afectada es el de Santa M aría, cuya deforestación desde 1983 (año en que fue considerada para el manejo debido a su mínima alteración y alta densidad poblacional hasta 1991 alcanzó el 51\% del área total.

EI incremento de la deforestación tiene mucho que ver con la tala y desbroce, practicadas durante las capturas. Estas deforestaciones parciales fueron aprovechadas y ampliadas principalmente en las restingas" de Santa M aría y Tahuayo. En río Tahuayo, las partes deforestadas corresponden a restingas bajas sujetas e inundaciones temporales de aguas negras, en consecuencia, los suelos son pobres, los mismos que tienen repercusiones en la baja producción del plátano y yuca. La vegetación secundaria en las chacras abandonadas o en proceso de empurme, están mayormente conformadas por ceticales (asociaciones de Cecropia spp.), y retamales (asociaciones de Cassia sp.) que por su escasa altura todavía no son utilizados por los A otus.

En Santa M aría, el área de estudio incluye alrededor de $70 \%$ de restinga baja, que a diferencia del Tahuayo es inundado temporalmente por una mezcla de agua blanca y 
negra, lo que determina un suelo mucho más fértil. De allí que gran parte de los cultivos lo conforman el arroz (O riza sativa) y maíz (zea maíz), así como frutales diversos, los mismos que en cierto modo favorecen a los Aotus, pues hacía ellas frecuentemente acuden para alimentarse de Guaba Psidium Guayaba) y caimito (Chrysophyllum cainito), entre otros.

\section{TABLA 5: Estimado de deforestación del bosque en las áreas de estudio con fines} agricolas.

\begin{tabular}{|c|c|c|c|}
\hline Af̆o & $\begin{array}{c}\text { Area Deforestada } \\
\text { (Has) }\end{array}$ & $\begin{array}{c}\% \\
\text { Incremento }\end{array}$ & $\begin{array}{c}\% \\
\text { del Area total }\end{array}$ \\
\hline $\begin{array}{c}\text { Tahuayo } \\
1987\end{array}$ & 0.0 & - & \\
1989 & 12.5 & 100.0 & \\
1991 & 22.5 & 80.0 & 11.2 \\
& & & \\
Tamboryacu & 3.6 & - & 6.0 \\
1987 & 6.0 & 66.0 & \\
1991 & & & \\
& 12.0 & - & \\
Santa María & 35.5 & 196.0 & \\
1983 & 51.0 & 33.0 & \\
1989 & & & \\
1991 & & & \\
\hline
\end{tabular}

\section{DISCUSION}

Los resultados de este estudio demuestran que las poblaciones después de la primera captura tanto de A. vociferans en Tamboryacu y de A. nancymae en Y anayacu se han recuperado satisfactoria, mientras que en otras como en Tahuayo y Santa M aría la recuperación es muy lenta. En el área de estudio de Tamboryacu, el potencial de recuperación queda demostrada para la población de A. vociferans, aun cuando nuestro resultado fue ligeramente inferior al nivel registrado en 1986, a pesar del tiempo suficientemente transcurrido. A quino et. al. (En prensa) demostró que para alcanzar el nivel de la población inicial en $A$. nancymae fue necesario entre 3 a 4 años, lo que no ha ocurrido en Tamboryacu cuyos factores desconocemos. En el área de estudio de Y anayacu, el potencial de recuperación de la población remanente es evidente. Estamos seguros que dentro de 2 a 3 años habrá al canzado el nivel

original a la primera captura. La recuperación es evidente, por cuanto hemos observado en casi todos los grupos la presencia de infantes, mucho de ellos serían el resultado de las migraciones de grupos completos de las áreas circundantes.

En el área de estudio del Tahuayo, el lento proceso de repoblación estaría relacionado a dos factores: a) La caza para subsistencia, y b) Deforestación con fines 
agrícolas. En Santa M aría, el factor afectante de mayor importancia es la deforestación con firmes agrícolas. Esta actividad en estos dos últimos años ha alcanzado niveles alarmantes, pues aproximadamente $51 \%$ del área de estudio fue destruida, causando serios perjuicios en el hábitat de A. vociferans, principalmente en lo concerniente al uso de huecos en los troncos y ramas de los árboles que fueron derribados para el propósito ya mencionado. Es indudable que los nacimientos e inmigraciones contribuyeron a la recuperación poblacional. La contribución de nacimientos fue confirmada por la presencia de infantes y juveniles durante la localización de los grupos familiares en Y anayacu y localización y captura en Tamboryacu. La contribución de inmigrantes puede ser argumentado por comparación del número de animales que permanecieron después de la primera captura (los cuales, dos años más tarde habrían sido adultos) con el número de adultos encontrados durante la última captura.

\section{CONCLUSIONES}

1. Las densidades poblacionales de A. vociferans y A. nancymae obtenidas demuestran que las capturas ejecutadas en las áreas de Tamboryacu y $Y$ anayacu no han tenido efectos negativos sobre la población. Contrariamente en Santa M aría y Tahuayo, las capturas desafortunadamente tuvieron efectos negativos sobre la población remanente.

2. Después de las capturas, los nacimientos y las inmigraciones contribuyeron a la recuperación de la población en Tamboryacu. Similares resultados vienen ocurriendo en $Y$ anayacu.

3. La alteración de la vegetación en las áreas de estudio de la quebrada Y anayacu y río Tamboryacu aparentemente no tienen efectos negativos sobre la población de Aotus, es más, la vegetación afectada viene recuperándose satisfactoriamente. En los ríos Santa María y Tahuayo; la tala para el aislamiento del árbol de dormir ha contribuido a una mayor deforestación ya que fueron convertidas en campos de cultivo.

\section{AGRADECIMIENTO}

$\mathrm{N}$ uestro agradecimiento para el personal especializado en capturas del Proyecto Peruano de Primatología y Caserío Huaysi en el río Tahuayo, quienes compartieron con nosotros gratas experiencias durante los trabajos de campo. Este estudio fue financiado por el Proyecto Peruano de Primatología (con fondos de la Carta de entendimiento M CP/ZN S/013 entre el Gobierno Peruano y la Organización Panamericana de la Salud) y la Universidad Nacional Mayor de San M arcos de Lima. 


\section{REFERENCIAS BIBLIOGRAFICAS}

Aquino, R; F. Encarnación, 1986. Population structure of Aotus nancymae Cebidae: Primates) in peruvian A mazon L owland Forest. AMERICAN JOURNAL OF PRIMATOLOGY 11: 1-7

A quino, R.; P. Puertas; F. Encarnación 1990. Suplemental noteson populations parametersof Northeastern peruvian night monkeys, genus Aotus (Cebidae). AMERICAN JOURNAL OF PRIMATOLOGY 21:215-221.

Aquino, R.;P. Puertas; F. Encarnación, Effects of cropping on the Aotus nancymae population in the forest of peruvian amazonia. AMERICAN JOURNAL OF PRIMATOLOGY (EN PRENSA).

Encarnación, E 1985. Introducción a la flora y vegetación de la amazonía peruana. Terminología y breve descripción de las formaciones vegetales de la llanura amazónica. CANDOLLEA 40: 237-252.

Sioli, $\mathrm{H}$. Tropical rivers as expressions of their terrestrial environments, pp. 275288. In: TROPICAL ECOLOGICAL SY STEMS. F.B. Golley, E, Medina, eds. New Y ork, Springer Verlag. 